\title{
DISFONIA INFANTIL: HÁBITOS PREJUDICIAIS À VOZ DOS PAIS INTERFEREM NA SAÚDE VOCAL DE SEUS FILHOS?
}

\author{
Children dysphonia: do harmful vocal habits by parents \\ interfere in their children vocal health?
}

\author{
Carla Lucélia Bessani Paixão ${ }^{(1)}$, Kelly Cristina Alves Silvério (2), Ana Paula Berberian ${ }^{(3)}$, \\ Lucia Figueiredo Mourão(4), Jair Mendes Marques ${ }^{(5)}$
}

\section{RESUMO}

Objetivo: verificar hábitos prejudiciais à voz referidos por crianças disfônicas e por seus respectivos pais e mães e compará-los com dados obtidos de um grupo controle, constituído por crianças sem alterações vocais e seus respectivos pais e mães. Método: investigou-se 28 crianças disfônicas, com idade entre 6 e 12 anos e seus pais (Grupo de Estudo - GE) e 22 crianças sem alterações vocais, com a mesma faixa etária, e seus respectivos pais (Grupo Controle - GC). As vozes foram classificadas em "alteradas" e "não alteradas" por meio de análise perceptivo-auditiva com amostra da fala espontânea.Todos responderam a um questionário sobre fatores prejudiciais à voz. Para análise dos resultados foram aplicados os testes de comparação de duas proporções $(p \leq 0,05)$. Resultados: verificou-se que as crianças do GE apresentaram significantemente mais os hábitos: falar com esforço, falar sem descansar e imitar vozes. Os pais do GE relataram significantemente mais hábitos de: pigarrear, gritar, falar junto com os outros, além de viver em ambiente de fumantes. As mães do GE apresentaram significantemente mais hábitos de: falar com esforço, falar em ambiente ruidoso e falar muito rápido. Viver em ambiente familiar ruidoso foi significantemente mais relatado por crianças, pais e mães do GE quando comparados aos do GC. Conclusão: independentemente de fatores que possam justificar a disfonia, crianças disfônicas e seus pais e mães relataram maior ocorrência de hábitos prejudiciais à voz e viver em ambiente familiar ruidoso do que crianças sem alterações vocais e seus respectivos pais e mães.

DESCRITORES: Distúrbios da Voz; Criança; Relações Familiares; Hábitos; Disfonia

(1) Fonoaudióloga; Mestre pelo Programa de Pós-Graduação em Distúrbios da Comunicação da Universidade Tuiuti do Paraná - UTP, Curitiba/PR - Brasil.

(2) Fonoaudióloga; Docente do Departamento de Fonoaudiologia da Faculdade de Odontologia de Bauru - Universidade de São Paulo - FOB/USP, São Paulo/SP - Brasil; Doutora em Ciências pela Faculdade de Odontologia de Piracicaba da Universidade Estadual de Campinas - FOP/ UNICAMP.

(3) Fonoaudióloga; Docente do Programa de Mestrado e Doutorado em Distúrbios da Comunicação da Universidade Tuiuti do Paraná - UTP, Curitiba/PR - Brasil; Doutora em Historia pela Pontifícia Universidade Católica de São Paulo.

(4) Fonoaudióloga; Docente do Curso de Fonoaudiologia da Universidade Estadual de Campinas - UNICAMP, Campinas/SP; Doutora em Neurociências pela Universidade Federal de São Paulo - UNIFESP.

(5) Matemático; Docente do Programa de Mestrado e Doutorado em Distúrbios da Comunicação da Universidade Tuiuti do Paraná - UTP, Curitiba/PR - Brasil; Doutor em Ciências Geodésicas pela Universidade Federal do Paraná - UFPR.

Conflito de interesses: inexistente

\section{INTRODUÇÃO}

A produção vocal realizada com esforço, sem harmonia e que limita o indivíduo na transmissão de sua mensagem verbal e emocional diz respeito a um transtorno vocal denominado disfonia'. $\mathrm{Na}$ infância, a disfonia pode estar relacionada a diversos fatores: alterações funcionais; alterações organofuncionais, como lesões nodulares; alterações orgânicas congênitas, tumores e estenoses laríngeas de graus variados que incapacitam e trazem risco de vida ${ }^{2-8}$; fatores respiratórios, alérgicos $^{10}$, digestórios ${ }^{11}$ fatores ambientais e psicológicos ${ }^{4}$. Há autores que propõem que as causas da disfonia infantil possam ser divididas em quatro categorias: orgânica, mudanças orgânicas resultantes de abuso vocal, funcional e outros fatores contribuintes ao problema vocal ${ }^{12}$. 
A formação do padrão vocal infantil está intimamente ligada a modelos vocais presentes no ambiente em que as crianças vivem. Por essa razão, pais e demais membros da família têm papel fundamental nas condições vocais das crianças ${ }^{12,13}$, podendo, portanto, influenciar os quadros de disfonia infantil. Apesar de tal influência, estudos evidenciam que pais e educadores dão pouca importância às alterações vocais na infância ${ }^{14}$, o que pode estar relacionado à dificuldade dos mesmos identificarem e reconhecerem a disfonia, fatores de risco que podem acarretar tal quadro, bem como suas implicações para a criança ${ }^{10,15}$.

Em relação à disfonia infantil de origem funcional, resultante de abuso vocal, a quantidade de pessoas que compõe as famílias numerosas é citada como fator de risco, assim como o hábito de seus membros falarem em forte intensidade. Tais características familiares são apontadas como favoráveis ao desenvolvimento e à prática de hábitos vocais inadequados como gritar, falar forte, berrar, realizar vocalizações tensas, falar excessivamente, fazer fonação invertida, vocalização explosiva ou ataque vocal brusco, pigarrear e falar em ambiente ruidoso ${ }^{12,16,17}$.

Estudo recente acerca da prevalência da disfonia infantil e de fatores associados evidenciou uma relação significante entre crianças disfônicas e a declaração dos pais sobre ambiente ruidoso em casa ${ }^{10}$.

Considerando o pressuposto de que a família tem papel preponderante no processo de desenvolvimento do padrão vocal infantil e no desenvolvimento de alguns quadros de disfonia infantil ${ }^{13,16,18,19}$, são restritos os estudos que priorizem a análise dessa relação ${ }^{1,15,16,18}$, o que demonstra preocupação incipiente com a investigação dos hábitos prejudiciais à voz praticados pelos pais.

Assim, este estudo tem por objetivo verificar os hábitos prejudiciais à voz referidos por crianças com alterações vocais e por seus respectivos pais e mães e compará-los com dados obtidos de um grupo controle, constituído por crianças sem alterações vocais e seus respectivos pais e mães.

\section{MÉTODO}

\section{Tipo de pesquisa}

Esta pesquisa consiste em um estudo de campo, transversal, comparativo e quantitativo.

\section{Processo de Amostragem}

Para o desenvolvimento do presente estudo, foram entregues 700 solicitações de autorização aos pais de crianças entre 6 e 12 anos de uma escola pública de ensino fundamental da cidade de
Curitiba, para realização de triagem fonoaudiológica vocal e do registro vocal, com devidas explicações acerca do estudo em questão. Foram recebidas 330 autorizações e, portanto, realizadas, na escola em ambiente silecioso, triagem fonoaudiológica vocal e gravação da voz com esse mesmo número de crianças. Para o registro das vozes, foi utilizado um microfone Ultimate headset unidirecional condenser profissional, estéreo, da marca Plantronics áudio $90 \AA$; acoplado a um computador notebook Toshiba modelo A135-S4737; e do software Multi Speech MDVP da Kay Elemetrics. As crianças foram orientadas a falar espontaneamente por 30 segundos, respondendo a questões como: "O que você acha da sua voz?" e "Do que você gosta de brincar?", respirando naturalmente e no seu ritmo de fala, com pitch e loudness habituais.

A análise perceptivo-auditiva foi realizada por três fonoaudiólogas, especialistas em voz, por consenso, com amostra da fala espontânea e consistiu da classificação das vozes em alteradas e não alteradas. Foram detectadas 58 crianças com algum tipo de alteração vocal.

Desse total, foram incluídas no estudo crianças que não apresentaram alterações neurológicas, neuromusculares, surdez ou qualquer tipo de malformação e que não passaram por tratamento fonoaudiológico vocal ou cirurgia da laringe. Somado a esses critérios, foram incluídas crianças que conviviam com seus respectivos pais diariamente na mesma residência, sendo excluídas do estudo aquelas cujos pais eram separados, ou que viviam com avós e demais responsáveis. Estes dados foram extraídos por meio do relato dos pais das crianças e/ou responsáveis, os quais compareceram à escola e responderam a um questionário contendo perguntas sobre estes aspectos.

Além disso, adotou-se, ainda, como critério de exclusão, crianças com desequilíbrios ressonantais, por presença de gripes e resfriados, alergias de vias aéreas respiratórias, hipo ou hipernasalidade ou mesmo presença de soprosidade de grau leve, verificados pela avaliação vocal perceptivoauditiva realizada.

Aplicados os critérios de inclusão e de exclusão, 28 crianças com alterações vocais foram selecionadas para compor o Grupo de Estudo (GE) e 22 crianças foram selecionadas para o Grupo Controle (GC). Após essa etapa, foi realizado um contato com os pais para devolutiva sobre os resultados e dar continuidade à pesquisa. Cabe ressaltar que, no presente estudo, as crianças com alterações vocais, serão denominadas "disfônicas", apesar de não terem avaliação laringológica para confirmar tal denominação. 


\section{Amostra}

Fizeram parte do grupo de estudo (GE) 28 crianças disfônicas de ambos os sexos (15 meninos e 13 meninas) com idades entre 6 e 12 anos (média $=9,9 \pm 1,6$ anos), e seus respectivos pais (média de idade $=40,5 \pm 6,0$ anos) e mães (média de idade $=38,1 \pm 6,3$ anos), totalizando 84 indivíduos. Para compor o GC, foram selecionadas 22 crianças sem alterações vocais, de ambos os sexos (9 meninos e 13 meninas) com idade de 6 a 12 anos (média de idade $=9,6 \pm 1,9$ anos) e seus respectivos pais (média de idade $=39,5 \pm 7,1$ anos) $\mathrm{e}$ mães (média de idade $=36,2 \pm 6,7$ anos), totalizando 66 indivíduos.

\section{Coleta de dados}

Para coleta dos dados, os 150 sujeitos que participaram da pesquisa responderam a um questionário formulado com questões fechadas, referentes a fatores prejudiciais à voz nos últimos seis meses: a. hábitos prejudiciais à voz - tossir freqüentemente, pigarrear, falar alto (forte), gritar, falar com esforço, falar em ambiente ruidoso, falar sussurrado, falar sem respirar, falar enquanto inspira, falar muito rápido, falar junto com os outros ou sem descansar, imitar sons, vozes de personagens, de pessoas conhecidas ou de heróis, ingerir pouca água, prática de esportes com uso da voz, e b. características do ambiente - viver em ambiente familiar ruidoso, viver em ambiente de fumantes ${ }^{13}$ (Figura 1). É importante enfatizar que pais, mães e crianças responderam ao questionário separadamente e, portanto, sem observar as respostas uns dos outros. As crianças não alfabetizadas ou com dificuldade em entender qualquer questão, contaram com a ajuda dos pesquisadores que liam as questões para elas. Da mesma forma, os pais receberam ajuda dos pesquisadores para leitura ou qualquer interpretação de questões que suscitassem dúvidas.

\section{Aspectos Bioéticos}

Todos os pais e crianças foram informados acerca do objetivo da pesquisa e convidados a assinar o Termo de Consentimento Livre e Esclarecido previamente aprovado pelo Comitê de Ética em Pesquisa da Universidade Tuiuti do Paraná (Of. CEP-UTP n0029/2007).

\section{Análise estatísitca}

Os dados obtidos foram tabulados e tratados estatisticamente a partir da aplicação do teste de diferença de proporções (nível de significância $0,05)$ para comparar os fatores prejudiciais à voz referidos pelos sujeitos que compõem os grupos GE e GC.

\section{RESULTADOS}

Os resultados encontram-se nas Tabelas 1 a 3.

Verificou-se que as crianças do GE apresentaram significantemente maior número de referências aos hábitos de falar com esforço $(p=0,0148)$, falar sem descansar $(p=0,0315)$, imitar vozes $(p=0,0257)$ e viver em ambiente familiar ruidoso ( $p=0,0001$ ), quando comparadas com as crianças do GC (Tabela 1). Foi possível observar que os pais do GE relataram significantemente mais hábitos de pigarrear $(p=0,0123)$, gritar $(0,0315)$, falar junto com os outros $(p=0,0306)$, viver em ambiente familiar ruidoso $(p=0,0009)$, além de viver em ambiente de fumantes $(p=0,0009)$ do que os pais do GC (Tabela 2). As mães do GE informaram apresentar, significantemente, mais hábitos de falar com esforço $(p=0,0411)$, falar em ambiente ruidoso $(p=0,0105)$, falar muito rápido $(p=0,0315)$ e viver em ambiente familiar ruidoso $(p=0,0005)$, em relação às mães que compõem o GC (Tabela 3).

\section{DISCUSSÃO}

Hábitos vocais inadequados colaboram para o mau uso da voz e estão relacionados a falar com competição de ruído ambiental, rir ou chorar excessivamente, tossir, pigarrear constantemente, imitar outras vozes ou imitar ruídos de personagens extraterrestres, heróis de televisão, monstros, dentre outros $^{7,13,18,19}$. No presente estudo, falar com esforço e sem descansar, bem como imitar sons, vozes de personagens, de pessoas conhecidas ou de heróis foram os hábitos significantemente mais relatados pelas crianças do GE quando comparadas às crianças do GC (Tabela 1), fato que mostra a maior ocorrência de usos vocais incorretos por parte das crianças disfônicas o que, somado a outros fatores, pode acarretar 0 aparecimento de alterações vocais $4,7,17,21$

Pesquisas apontam que disfonias associadas, por exemplo, à formação de nódulos, podem ser atribuídas a abusos vocais, especialmente gritar, falar com esforço, falar em forte intensidade por períodos prolongados ${ }^{4,7,17,21}$. Da mesma forma, pode-se observar que crianças que possuem famílias mais numerosas e vivem em ambiente familiar ruidoso, costumam ter mais alterações vocais ${ }^{4,10}$. Estudo revela que crianças com irmãos mais velhos foram significantemente mais propensas a serem identificadas como tendo uma voz alterada por seus pais ${ }^{10}$. Falar com esforço e sem descansar, bem como falar em forte intensidade são hábitos vocais relacionados a uma dinâmica familiar que tende a promover ambiente ruidoso ${ }^{13}$. No presente estudo, o ambiente familiar ruidoso foi significantemente mais 


\section{Identificação:}

\begin{tabular}{|l|l|}
\hline \multicolumn{2}{|l|}{ Nome completo: } \\
\hline Data de nascimento: \\
\hline Outra atividade: & \multicolumn{2}{|l|}{} \\
\hline Mãe: & \multicolumn{2}{|l|}{} \\
\hline Outra atividade: & Profissão: \\
\hline Pai: & \multicolumn{2}{|l|}{} \\
\hline Outra atividade: & Profissão: \\
\hline Irmão(s): & Idade: \\
\hline Moradores da casa: & \multicolumn{2}{|l|}{} \\
\hline Endereço: & \\
\hline Telefone para contato: & \\
\hline
\end{tabular}

\section{Assinale com um " $x$ " os hábitos que você apresenta há pelo menos 6 meses:}

\section{Fatores relacionados à voz}

( ) Tossir frequentemente

( ) Pigarrear

( ) Falar alto (forte)

( ) Gritar

( ) Falar com esforço

( ) Falar em ambientes ruidosos

( ) Falar sussurrado

( ) Falar sem respirar

( ) Falar enquanto inspira (puxa o ar)

( ) Falar muito rápido

( ) Falar junto com os outros

( ) Falar sem descansar

( ) Imitar sons, vozes de personagens, de pessoas conhecidas ou de heróis

( ) Ingerir pouca água

( ) Pratica esportes que necessitam do uso da voz

( ) Fumo - cigarros/dia há quanto tempo

( ) Álcool-dose/dia há quanto tempo

\section{Fatores relacionados ao ambiente familiar}

( )Vive em ambiente familiar ruidoso

( )Vive em ambiente de fumantes

\section{Figura 1 - Questionário sobre hábitos prejudiciais à voz}

relatado pelas crianças, pais e mães pertencentes ao GE, quando comparados com os integrantes do GC (Tabelas 1, 2 e 3). Este fato evidencia que o ambiente ruidoso, recorrentemente mencionado pelos sujeitos da pesquisa, é uma das características familiares das crianças disfônicas do grupo estudado. Ressalta-se, ainda que as mães do GE relataram significantemente mais o hábito de falar em ambiente ruidoso em relação às mães do GC.

O hábito de gritar foi relatado na presente pesquisa por $75 \%$ das crianças do GE e $50 \%$ das crianças do GC (Tabela 1). Verifica-se que este é um hábito muito praticado por crianças disfônicas ou não e que, portanto, não deve ser considerado isoladamente como fator determinante no desenvolvimento de alterações vocais. Vários autores afirmaram que crianças com nódulos vocais são geralmente caracterizadas como ativas e que costumam fazer frequentemente uso do grito $4,12,13,21,22$, dentre outros comportamentos vocais abusivos. 
Tabela 1 - Distribuição de crianças do GE $\left(n_{1}=28\right)$ e GC $\left(n_{2}=22\right)$, em números absolutos e porcentagem, e valor de $\mathrm{p}$, em relação aos fatores prejudiciais à voz

\begin{tabular}{lccc}
\hline Fatores prejudiciais à voz & $\begin{array}{c}\text { Crianças Grupo } \\
\text { de Estudo }\end{array}$ & $\begin{array}{c}\text { Crianças Grupo } \\
\text { Controle }\end{array}$ & Valor de p \\
\hline Tossir frequentemente & $7(25,0 \%)$ & $5(22,7 \%)$ & 0,8703 \\
Pigarrear & $12(42,9 \%)$ & $5(22,7 \%)$ & 0,1455 \\
Falar alto (forte) & $17(69,7 \%)$ & $14(63,6 \%)$ & 0,6564 \\
Gritar & $21(75,0 \%)$ & $11(50 \%)$ & 0,0738 \\
Falar com esforço & $7(25,0 \%)$ & $0(0 \%)$ & ${ }^{*} \mathbf{0 , 0 1 4 8}$ \\
Falar em ambiente ruidoso & $9(32,1 \%)$ & $6(27,3 \%)$ & 0,7029 \\
Falar sussurrado & $2(7,1 \%)$ & $1(4,5 \%)$ & 0,7112 \\
Falar sem respirar & $7(25,0 \%)$ & $6(27,3 \%)$ & 0,8733 \\
Falar enquanto inspira & $9(32,1 \%)$ & $4(18,2 \%)$ & 0,2672 \\
Falar muito rápido & $11(39,3 \%)$ & $10(45,5 \%)$ & 0,6210 \\
Falar junto com outros & $16(57,1 \%)$ & $11(50 \%)$ & 0,6243 \\
Falar sem descansar & $10(35,7 \%)$ & $2(9,1 \%)$ & ${ }^{\mathbf{0}, 0315}$ \\
Imitar sons, vozes de personagens, & $21(75,0 \%)$ & $12(42,9 \%)$ & ${ }^{*} \mathbf{0 , 0 2 5 7}$ \\
de pessoas conhecidas ou de heróis & $15(53,6 \%)$ & $13(59,1 \%)$ & 0,7251 \\
Ingerir pouca água & $11(39,3 \%)$ & $6(27,3 \%)$ & 0,3774 \\
Prática de esportes com uso da voz & $23(82,1 \%)$ & $5(22,7 \%)$ & ${ }^{*} \mathbf{0 , 0 0 0 1}$ \\
Viver em ambiente familiar ruidoso & $2(7,1 \%)$ & $4(18,2 \%)$ & 0,2379 \\
Viver em ambiente de fumantes & & &
\end{tabular}

${ }^{*} \mathrm{p}<0,05-$ teste de diferença de proporções

Tabela 2 - Distribuição de pais do GE $\left(n_{1}=28\right)$ e GC $\left(n_{2}=22\right)$, em números absolutos e porcentagem, e valor de $p$, em relação aos fatores prejudiciais à voz

\begin{tabular}{lccc}
\hline Fatores prejudiciais à voz & $\begin{array}{c}\text { Pai } \\
\text { Grupo de Estudo }\end{array}$ & $\begin{array}{c}\text { Pai } \\
\text { Grupo Controle }\end{array}$ & Valor de p \\
\hline Tossir frequentemente & $5(17,9 \%)$ & $3(13,6 \%)$ & 0,7051 \\
Pigarrear & $15(53,6 \%)$ & $4(18,2 \%)$ & ${ }^{*} \mathbf{0 , 0 1 2 3}$ \\
Falar alto (forte) & $14(50 \%)$ & $6(27,3 \%)$ & 0,1057 \\
Gritar & $10(35,7 \%)$ & $2(9,1 \%)$ & ${ }^{*} \mathbf{0 , 0 3 1 5}$ \\
Falar com esforço & $3(10,7 \%)$ & $0(0 \%)$ & 0,1149 \\
Falar em ambiente ruidoso & $11(39,3 \%)$ & $4(18,2 \%)$ & 0,1135 \\
Falar sussurrado & $2(7,1 \%)$ & $0(0 \%)$ & 0,2116 \\
Falar sem respirar & $6(21,4 \%)$ & $2(9,1 \%)$ & 0,2529 \\
Falar enquanto inspira & $2(7,1 \%)$ & $2(9,1 \%)$ & 0,7956 \\
Falar muito rápido & $10(35,7 \%)$ & $5(22,7 \%)$ & 0,3256 \\
Falar junto com outros & $8(28,6 \%)$ & $1(4,5 \%)$ & $* \mathbf{0 , 0 3 0 6}$ \\
Falar sem descansar & $4(14,3 \%)$ & $2(9,1 \%)$ & 0,5890 \\
Imitar sons, vozes de personagens, & $8(28,6 \%)$ & $3(13,6 \%)$ & 0,2128 \\
de pessoas conhecidas ou de heróis & $16(57,1 \%)$ & $11(50 \%)$ & 0,6243 \\
Ingerir pouca água & $3(10,7 \%)$ & $0(0 \%)$ & 0,4167 \\
Prática de esportes com uso da voz & $5(17,9 \%)$ & $2(9,1 \%)$ & 0,3677 \\
Fumo & $10(35,7 \%)$ & $6(27,3 \%)$ & 0,5017 \\
Álcool & $19(67,9 \%)$ & $2(9,1 \%)$ & $* \mathbf{0 , 0 0 0 1}$ \\
Viver em ambiente familiar ruidoso & $12(42,9 \%)$ & $0(0 \%)$ & $* \mathbf{0 , 0 0 0 9}$ \\
Viver em ambiente de fumantes & & & \\
\hline
\end{tabular}

${ }^{*} \mathrm{p}<0,05$ - teste de diferença de proporções 
Tabela 3 - Distribuição de mães do GE $\left(n_{1}=28\right)$ e GC $\left(n_{2}=22\right)$, em números absolutos e porcentagem, e valor de $p$, em relação aos fatores prejudiciais à voz

\begin{tabular}{lccc}
\hline Fatores prejudiciais à voz & $\begin{array}{c}\text { Mães } \\
\text { Grupo de Estudo }\end{array}$ & $\begin{array}{c}\text { Mães } \\
\text { Grupo Controle }\end{array}$ & Valor de p \\
\hline Tossir frequentemente & $0(0 \%)$ & $1(4,5 \%)$ & 0,2625 \\
Pigarro & $8(28,6 \%)$ & $2(9,1 \%)$ & 0,0868 \\
Falar alto (forte) & $14(50 \%)$ & $7(31,8 \%)$ & 0,2068 \\
Gritar & $13(46,2 \%)$ & $5(22,7 \%)$ & 0,0988 \\
Falar com esforço & $5(17,9 \%)$ & $0(0 \%)$ & $* \mathbf{0 , 0 4 1 1}$ \\
Falar em ambiente ruidoso & $10(35,7 \%)$ & $1(4,5 \%)$ & $* \mathbf{0 , 0 1 0 5}$ \\
Falar sussurrado & $0(0 \%)$ & $0(0 \%)$ & - \\
Falar sem respirar & $3(10,7 \%)$ & $1(4,5 \%)$ & 0,3675 \\
Falar enquanto inspira & $2(7,1 \%)$ & $0(0 \%)$ & 0,2116 \\
Falar muito rápido & $10(35,7 \%)$ & $2(9,1 \%)$ & $* \mathbf{0 , 0 3 1 5}$ \\
Falar junto com outros & $5(17,9 \%)$ & $2(9,1 \%)$ & 0,3677 \\
Falar sem descansar & $5(17,9 \%)$ & $2(9,1 \%)$ & 0,3677 \\
Imitar sons, vozes de personagens, & $1(3,6 \%)$ & $0(0 \%)$ & 0,3475 \\
de pessoas conhecidas ou de heróis & $15(53,6 \%)$ & $9(40,9 \%)$ & 0,3657 \\
Ingerir pouca água & $0(0 \%)$ & $0(0 \%)$ & - \\
Prática de esportes com uso da voz & $3(10,7 \%)$ & $2(9,1 \%)$ & 0,8169 \\
Fumo & $3(10,7 \%)$ & $4(18,2 \%)$ & 0,4834 \\
Álcool & $15(53,6 \%)$ & $1(4,5 \%)$ & $* \mathbf{0 , 0 0 0 5}$ \\
Viver em ambiente familiar ruidoso & $5(17,9 \%)$ & $1(4,5 \%)$ & 0,1522 \\
Viver em ambiente de fumantes & & &
\end{tabular}

${ }^{*} p<0,05$ - teste de diferença de proporções

É importante esclarecer que considerar apenas o abuso vocal da criança - seja ele qual for - como fator etiológico do aparecimento de nódulos vocais, pode revelar uma abordagem reducionista, frente à multiplicidade dos fatores etiológicos do aparecimento desse tipo de lesão na infância ${ }^{4,7,12}$. Enfim, fatores orgânicos ${ }^{2,7,11}$, emocionais ${ }^{4,13}$ e ambientais ${ }^{7,13}$ são apontados como relevantes na constituição de tais quadros, embora os usos incorretos da voz estejam presentes na maioria das alterações vocais nas crianças ${ }^{7,15,17,22}$.

Nesse sentido, dentre os fatores ambientais que propiciam usos vocais incorretos, há que se considerar modelos familiares vocais inadequados, presentes no contexto em que a criança está inserida, como facilitadores do surgimento de alterações vocais na infância. Entende-se que no sistema familiar o padrão de fala de cada indivíduo influencia e é influenciado pelos demais ${ }^{23}$.

Desta forma, pais que fazem uso recorrente do grito com a finalidade de chamar e/ou conversar com outras pessoas, emitir ordens, transmitir informações, oferecem modelos vocais que podem ser reconhecidos e incorporados pela criança como eficientes para resolver parte das necessidades do dia-a-dia ${ }^{13,16}$.

Quanto a esse aspecto, pôde-se constatar, no presente estudo, que os pais das crianças disfônicas (GE) declararam praticar significantemente mais os hábitos de gritar e falar junto com os outros do que os pais das crianças sem alterações vocais (GC). Somado a esse fato, as mães pertencentes ao GE declararam falar com esforço, falar muito rápido e em ambiente ruidoso significantemente mais do que as mães do GC. Embora o hábito de gritar também tenha sido maior nas mães do GE $(46,2 \%)$ quando comparadas às mães do GC $(22,7 \%)$, mas essa diferença não foi significante. É possível sugerir a influência do hábito prejudicial à voz das mães - falar com esforço - sobre as crianças do GE, já que elas declararam a presença do mesmo hábito significantemente mais do que as crianças do GC.

Além dos hábitos prejudiciais à voz, os pais do GE referiram, ainda, número significante de outros hábitos inadequados, quando comparados aos pais das crianças do GC, como pigarrear e viver em ambientes de fumantes. Embora pigarrear possa estar relacionado a outros fatores como refluxo 
gastresofágico ou alergias, quando somado aos demais hábitos relatados pelos pais, podem representar risco à saúde vocal. Os dados obtidos neste estudo reiteram o pressuposto de que modelos vocais e de saúde inadequados adotados frequentemente por pais e mães de crianças disfônicas (GE) são indicadores de risco que podem causar, perpetuar ou agravar quadros de disfonia. Desta forma, investigar a família quanto ao uso da voz, ao hábito de gritar ou de falar forte em diferentes situações, aos conhecimentos acerca dos sintomas que caracterizam alterações vocais, ao nível de ruído em casa, ou mesmo do que cada um diz sobre sua própria voz pode oferecer elementos reveladores da dinâmica familiar frente à disfonia infantil 13,24-25.

A ocorrência simultânea de padrões inadequados como falar com esforço e sem descansar, gritar, imitar vozes e conviver em ambiente familiar ruidoso é favorável ao aparecimento da alteração vocal em crianças que apresentam alguma predisposição ${ }^{22}$. Imitar sons, vozes de personagens, de pessoas conhecidas ou de heróis foi declarado, com significância estatística, por $75 \%$ das crianças disfônicas (GE) contra 42,9\% das crianças não disfônicas (GC) (Tabela 1). Esses dados indicam, ainda, que, embora crianças sem alterações vocais também imitem vozes e gritem, o fato de não conviverem em ambiente familiar ruidoso e com familiares que mantêm um modelo vocal abusivo, parece diminuir as chances de desenvolverem a disfonia.

Se estudos preconizam que o relacionamento da criança disfônica com sua família deve ser abordado durante o processo terapêutico fonoaudiológico ${ }^{9,13,17-18}$, este trabalho enfatiza a importância da família desde o momento do diagnóstico, uma vez que ter acesso a características da dinâmica familiar, no que se refere a padrões de comunicação e do ambiente, é fundamental para a definição do diagnóstico, bem como para o planejamento e desenvolvimento de medidas de reeducação vocal que envolvam não só a criança, como seus familiares.

A maior ocorrência de hábitos prejudiciais à voz por parte de pais e mães de crianças disfônicas evidencia que modelos vocais familiares inadequados constituem-se como um dos fatores de risco para o desenvolvimento de disfonias. Esse fato reforça a necessidade da implementação de estudos que analisem as implicações de tais hábitos na saúde vocal, priorizando a correlação da prática dos mesmos por crianças e por seus familiares.

\section{CONCLUSÃO}

Os dados encontrados neste estudo permitem concluir que independentemente de fatores que possam justificar a disfonia, os fatores prejudiciais à voz praticados pelas crianças disfônicas, bem como seus pais e mães são maiores do que pelas crianças sem alterações vocais e seus respectivos pais e mães. Pode-se evidenciar, enquanto fator de risco à saúde vocal, que viver em ambiente familiar ruidoso é significantemente mais referido pelas crianças disfônicas e seus pais.

Este estudo reforça a necessidade de se considerar durante todo o processo terapêutico os fatores prejudiciais à voz praticados pelos pais e não somente pelas crianças, além de se investigar o ruído no ambiente em que as crianças vivem, minimizando os riscos de surgimento, manutenção e recorrência das disfonias. 


\section{ABSTRACT}

Purpose: to investigate harmful vocal habits referred by dysphonic children and their parents and compare them with data gathered from a control group made up by children with no vocal alterations and their parents. Method: twenty-eight dysphonic children, with ages between 6 and 12 year old and their parents, (Study Group - SG) along with 22 children with no vocal alterations, from the same age group, and their parents (Control Group - CG) were investigated. The voices were classified as "healthy" and "unhealthy", depending on the result obtained from the percentage analysis of a spontaneous speech sample. Subjects answered a questionnaire about harmful vocal habits. Tests for comparing two proportions $(p<0.05)$ were used in order to analyze the results. Results: SG children showed a significantly higher number of habits such as speaking with effort, speaking without resting and imitating voices. SG fathers showed a significantly higher number of habits such as clearing the throat, shouting, speaking at the same time as others, in addition to living in smokers' environment. SG mothers significantly showed more habits such as speaking with effort, speaking in noisy environments and speaking too quickly. Living in noisy familiar environments was significantly reported by children, fathers and mothers of SG when compared to those of the CG. Conclusion: despite the factors that explain dysphonia, dysphonic children as well as their fathers and mothers reported a higher incidence of harmful vocal habits and living in noisy familiar environments than children with no vocal alterations and their parents.

KEYWORDS: Voice Disorders; Child; Family Relations; Habits; Dysphonia

\section{REFERÊNCIAS}

1. Teixeira MZM, Trezza EMC, Behlau M. Opinião dos pais sobre a voz de seus filhos de 5 a 12 anos. Rev. Paul Pediatria. 2003; 21(2): 68-75.

2. Nicollas R, Giovanni A, Triglia JM. Dysphonia in children. Arch Pediatr. 2008 Jun;15(6):1133-8.

3. Trani M, Ghidini A, Bergamini G, Presutti L. Voice therapy in pediatric functional dysphonia: a prospective study. Int J Pediatr Otorhinolaryngol. 2007 Mar;71(3):379-84.

4. Roy N, Holt KI, Redmond S, Muntz H. Behavioral characteristics of children with vocal fold nodules. J Voice. 2007; 21(2): 157-68.

5. Merati AL, Keppel K, Braun NM, Blumin JH, Kerschner JE. Pediatric voice-related quality of life: findings in healthy children and in common laryngeal disorders. Ann Otol Rhinol Laryngol. 2008; 117(4): 259-62.

6. Tavares EL; Labio RB; Martins RH. Normative study of vocal acoustic parameters from children from 4 to 12 years of age without vocal symptoms: a pilot study. Braz J Otorhinolaryngol. 2010;76(4): 485-90.

7. Connelly A, Clement WA, Kubba H. Management of dysphonia in children. J Laryngol Otol. 2009;123(6): 642-7.

8. Mortensen M, Schaberg M, Woo P. Diagnostic Contributions of Videolaryngostroboscopy in the
Pediatric Population. Arch Otolaryngol Head Neck Surg. 2010;136 (1):75-9.

9. Schindler A, Capaccio P, Maruzzi P, Ginocchio D, Bottero A, Otraviani F. Preliminary considerations on the application of the voice handicap index to paediatric dysphonia. Acta Otorhinolaryngol Ital. 2007 Feb;27(1):22-6.

10. Carding PN, Roulstone S, Northstone K, ALSPAC Study Team. The prevalence of childhood dysphonia: a cross-sectonal study. J Voice. 2006; 20(4): 623-30.

11. Block B, Brodsky L. Hoarseness in children: the role of laryngopharyngeal reflux. Int $\mathrm{J}$ Pediatr Otorhinolaryngol. 2007; 71 (9):1361-9.

12. Maia AA, Gama ACC, Michalick-Triginelli MF. Relação entre transtorno do déficit de atenção/ hiperatividade, dinâmica familiar, Disfonia e nódulo vocal em crianças. Rev. Ciênc. Méd. 2006; 15(5):379-89.

13. Azevedo R. Disfonia na Infância. In: Fernandes FDM, Mendes BCA, Navas ALPGP (org). Tratado de Fonoaudiologia. 2 $2^{\underline{a}}$ ed. São Paulo: Ed. Roca; 2010. 709-14.

14. Fuchs M, Meuret S, Stuhrmann NC, Schade G. Dysphonia in children and adolescents. HNO. 2009 Jun;57(6):603-14.

15. Takeshita TK, Aguiar-Ricz L, Isaac ML, Ricz H, Anselmo-Lima W. Comportamento vocal de crianças em idade pré-escolar. Arq. Int. Otorrinolaringol. 2009; 13(3):252-8. 
16. Wilson DK. Problemas de voz em crianças. São Paulo: Manole; 1993.

17. Tezcaner CZ, Ozgursoy SK, Sati I, Dursun G. Changes after voice therapy in objective and subjective voice measurements of pediatric patients with vocal nodules. Eur Arch Otorhinolaryngol. 2009 Dec;266(12):1923-7.

18. Behlau MS, Gonçalves MIR. Considerações sobre a disfonia infantil. In: Ferreira LP. Trabalhando com a voz. São Paulo: Summus Editorial; 1988. 99-107.

19. Kyrillos LCR. Distúrbios da voz em crianças: fatores causais e prevenção. Rev. O Mundo da Saúde. 1995; 19(5):177-81.

20. Angelillo N, Di Costanzo B, Angelillo M, Costa G, Barillari MR, Barillari U. Epidemiological study on vocal disorders in pediatric age. J Prev Med Hyg. 2008 Mar;49(1):1-5.

21. Akif KM, Okur E, Yildirim I, Güzelsoy S. The prevalence of vocal fold nodules in school age children. Int J Pediatr Otorhinolaryngol. 2004; 68(4): 409-12.

22. Andrade CRF, Sepulcre AS, Romano MVR, Juste F, Sassi C. Percepção de pais de crianças gagas e fluentes sobre as características de temperamento de seus filhos. Rev Soc Bras Fonoaudiol. 2004; 9(4):205-11.

23. Blumin JH, Keppel KL, Braun NM, Kerschner JE, Merati AL. The impact of gender and age on voice related quality of life in children: normative data. Int J Pediatr Otorhinolaryngol. 2008;72(2):229-34 .

24. Connor NP, Cohen SB, Theis SM, Thiebeault SL, Heatley DG, Bless DM.

Attitudes of children with dysphonia. J Voice. 2008;22(2):197-209.

25. Verduyckt I, Remacle M, Jamart J, Benderitter C, Morsomme D. Voice-related complaints in the pediatric population. J Voice. 2010. Article in press. doi:10.1016/j.jvoice.2009.11.008.
http://dx.doi.org/10.1590/S1516-18462011005000116

RECEBIDO EM: 28/03/2011

ACEITO EM: 17/05/2011

Endereço para correspondência:

Kelly Cristina Alves Silvério

Departamento de Fonoaudiologia

Al. Dr. Octávio Pinheiro Brisolla, 9-75

Bauru - SP

CEP: $17012-901$ - C.P. 73

E-mail: kcsilver@terra.com.br

kellysilverio@usp.br 